\title{
Un CONJUNTO DE CERÁMICAS ÁTICAS INÉDITAS DE LA NECRÓPOLIS DE Olival do Senhor dos Mártires (Alcácer do Sal, Portugal)
}

A new ensemble of attic pottery from the Necropolis of Olival do Senhor dos Mártires (Alcácer do Sal, Portugal)

Francisco B. Gomes UNIARQ - Centro de Arqueologia da Universidade de Lisboa

Recibido: 10/01/2017

Revisado: 01/03/2017
Aceptado: $19 / 05 / 2017$

Publicado: 23/06/2017

\section{RESUMEN}

Se presenta un conjunto de ocho fragmentos de cerámica ática inéditos provenientes de la necrópolis de Olival do Senhor dos Mártires (Alcácer do Sal, Portugal). Como forma de encuadrar estos nuevos materiales, se analiza la posición de los materiales griegos del yacimiento en la dinámica general del comercio griego con el actual territorio portugués, y bien así su significado cultural y cronológico en el marco de la secuencia de utilización de la necrópolis.

\section{Palabras Clave}

Olival do Senhor dos Mártires (Alcácer do Sal, Portugal); II Edad del Hierro; cerámica ática; figuras rojas; barniz negro.
ABSTRACT

This paper aims to present a previously unpublished ensemble of eight fragments of attic pottery from the necropolis of Olival do Senhor dos Mártires (Alcácer do Sal, Portugal). Taking this material as a starting point, it also addresses the position of this site in the overall framework of the Greek trade with the currently Portuguese territory and particularly the cultural and chronological significance of the Greek material for the analysis of the necropolis's sequence.

\section{KEY WORDS}

Olival do Senhor dos Mártires (Alcácer do Sal, Portugal); Late Iron Age; Attic pottery; red figures; black glaze.

franciscojbgomes@gmail.com 
1. La necrópolis de Olival do SENHOr dos Mártires (Alcácer do Sal, Portugal): Breve introducCIÓN.

Situada sensiblemente un kilómetro al Occidente de la elevación donde se erigió el castillo medieval de Alcácer do Sal (Fig. 1), la necrópolis de Olival do Senhor dos Mártires (OSM) es conocida desde el siglo XIX cuando la realización de trabajos agrícolas resultó en el descubrimiento de un importante conjunto de materiales, dados a conocer en seguida por J. N. Possidónio da Silva (1875), entre los cuales se contaba un conjunto de recipientes de producción ática que, como adelante se verá, han llamado fuertemente la atención de la comunidad investigadora.

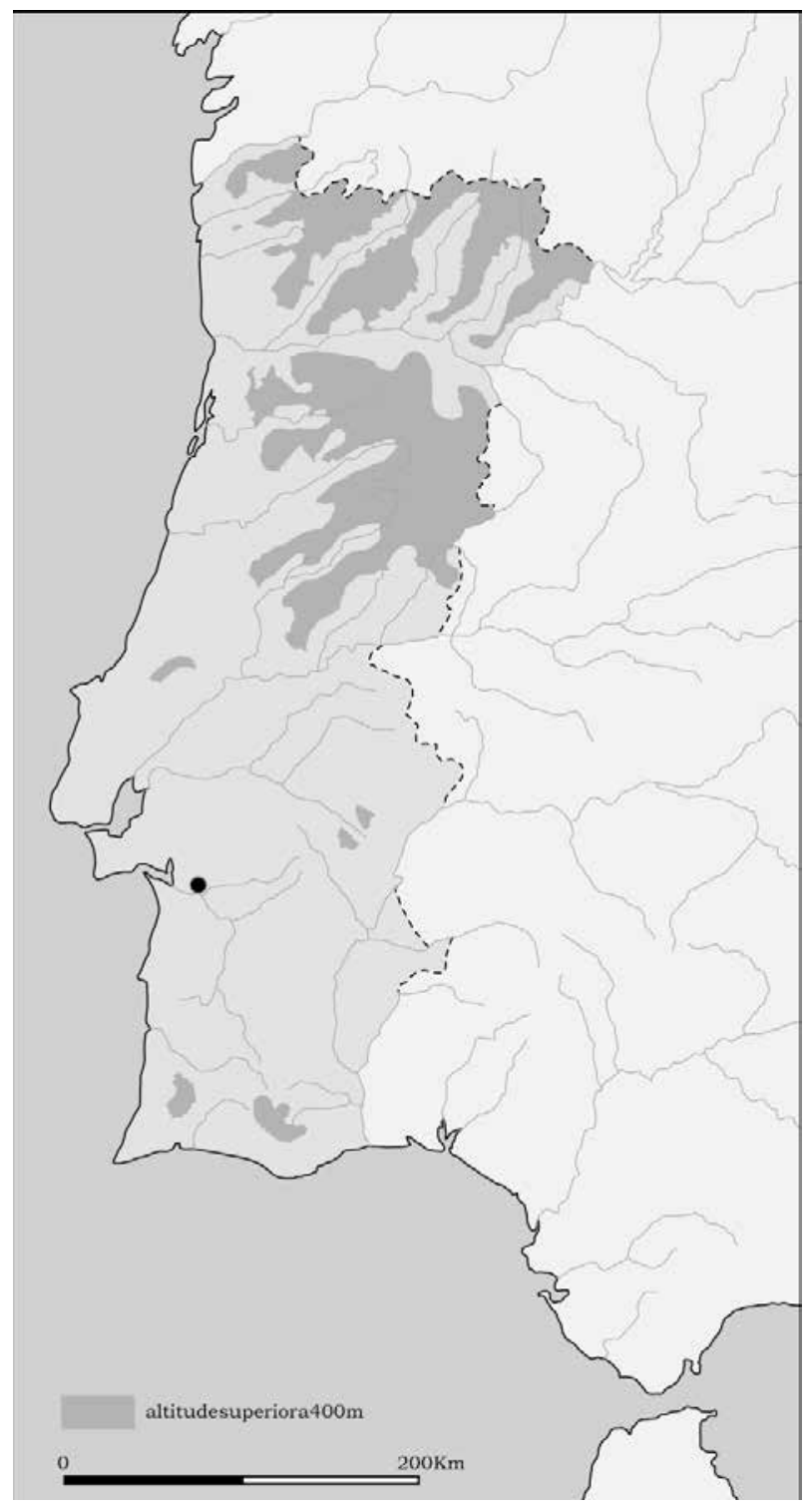

Figura 1. Ubicación de la necrópolis de Olival do Senhor dos Mártires en el actual territorio portugués.
A pesar del interés inmediato provocado por estos primeros hallazgos, debido a un conflicto administrativo entre el Estado Portugués y el propietario de los terrenos donde se ubicaba la necrópolis el yacimiento no fue objeto de trabajos sistemáticos hasta mucho más tarde; de hecho, las primeras excavaciones sólo tendrían lugar ya en los años 1920 bajo la dirección del Profesor V. Correia, quien llevó a cabo allí una serie de campañas de excavaciones, desgraciadamente nunca publicadas en profundidad.

Aun así, las breves notas y artículos que sí produjo sobre sus trabajos en este conjunto funerario (Correia 1925a, 1925b, 1925c, 1928 y 1930) son sumamente iluminantes, y han permitido por primera vez conocer con cierto grado de fiabilidad el tipo de estructuras sepulcrales que lo componen, los rituales funerarios y algunos aspectos de los propios ajuares y de su articulación.

En las décadas siguientes, el conocimiento sobre la necrópolis se fue incrementando con la realización de estudios que, a pesar de significativos y relevantes, se revistieron siempre de un carácter muy parcial y selectivo (Gomes, 2015). Los trabajos de campo en la necrópolis no se reanudarían, no obstante, hasta los años 1960; en el final de esta década tuvieron lugar nuevas excavaciones en el yacimiento bajo la dirección de A. Cavaleiro Paixão, cuyos resultados fueron sistemáticamente recogidos en su tesis de licenciatura (Paixão, 1970 y 2014).

El mismo investigador realizó, entre finales de la década de 1970 e inicios de la de 1980, nuevas intervenciones en la necrópolis, de las cuales, no obstante, se sabe muy poco, ya que sólo se publicó de forma desarrollada una nota sobre una de las tumbas excavadas (Paixão, 1983).

Por otro lado, y también en este momento, la publicación de los datos resultantes de un sondeo realizado en el cerro del castillo permitió por primera vez valorar la secuencia cultural del poblado al que está asociada la necrópolis del OSM, documentando una importante ocupación de la Edad del Hierro (Silva et al., 1980,1).

En los años subsecuentes vieron la luz nuevos trabajos dedicados al estudio de ciertas categorías materiales, entre los que cabría destacar, en este contexto, el primer estudio integral de la cerámica griega de la necrópolis (Rouillard et al., 1988, 9). Finalmente, y ya a finales de los años 1990, se produjeron dos importantes síntesis que, mediante el análisis de la información publicada hasta entonces, permitieron abordar por primera vez de forma 
sistemática y actualizada la secuencia cultural de la necrópolis (Fabião, 1998, 350-366; Arruda, 19992000, 72-86), sentando en buena medida las bases del conocimiento actual sobre la necrópolis.

No es esta la ocasión más adecuada para reseñar el cuadro general de referencia resultante de este historial de investigación, que por otra parte ya he buscado resumir en otros contextos (Gomes, 2015, 2016), pero parece indispensable recordar brevemente los datos disponibles sobre el horizonte de la II Edad del Hierro del OSM, en el cual se enmarcan los materiales estudiados en esta contribución.

Aparte de los materiales conocidos de este período, la valoración de dicho horizonte se basa únicamente en los testimonios de V. Correia, que ha podido excavar un conjunto de tumbas fechables en este momento, hecho que no se repetirá en las intervenciones posteriores, en las que sólo se documentaron contextos de la I Edad del Hierro.

Estas deposiciones, encuadradas por el Profesor de Coimbra en el $1^{\text {er }}$ Tipo de su tipología de tumbas (Correia, 1928), corresponden a incineraciones con deposición secundaria de los restos cremados en contenedores cinerarios, depositados a su vez en sencillas fosas excavadas en el terreno, desprovistas de cualquier superestructura compleja (ibídem). Dichos contenedores parecen haber sido de distintas tipologías, incluyendo krateres griegos (cf. infra), pero también urnas bi-troncocónicas de tipo “Turdetano” (Frankenstein, 1997, Láms. 53-4 y 56), aparte posiblemente de otros tipos de recipientes menos característicos.

Las deposiciones se acompañaban de ajuares muy diversos, incluyendo, siempre de acuerdo con el testimonio de V. Correia, “...um ou dois vasos pequenos (...) e as duas coçoiras ou fusaïolas rituais. Por baixo da urna amontoavam-se (...) as armas e os adereços do defunto: - enovelado o soliferreum, dobradas as laminas das falcatas e das adagas, encurvadas as folhas das lanças longas, deformadas as baínhas, torcidas as placas de cinturão, as fíbulas, os braceletes" (Correia, 1928, 173).

Como ya se ha discutido en distintas ocasiones (Fabião, 1998, 350-366; Arruda, 1999-2000, 73) y yo mismo he podido comentar en otros trabajos (Gomes, 2015 y 2016), la mayoría de estos elementos se pueden identificar con cierta seguridad entre el material publicado, con lo cual la definición de los contenidos materiales de este horizonte tardío dentro de la secuencia de la necrópolis se revela más factible que la de los de las fases más antiguas.
Aun así, siguen existiendo problemas sustanciales en el análisis de esta etapa, que he buscado sanar con el estudio monográfico de los materiales (Gomes, 2016) pero que no siempre se pueden, hoy por hoy, considerar resueltos. Así, por ejemplo, el proceso de transición entre las soluciones funerarias más antiguas y las que caracterizan la II Edad del Hierro sigue siendo una cuestión a debate, que sólo un mejor conocimiento de los estratos correspondientes del poblado podrá contestar de forma más resolutiva.

La propia duración y secuencia interna de la fase prerromana de la necrópolis no se encuentra, hoy por hoy, debidamente caracterizada, hecho a que no es ajena la escasa definición cronológica de los materiales de este período, con lo cual la vajilla griega, que sí se puede fechar de forma muy precisa, acabó teniendo un peso desproporcional en el establecimiento de la cronología global de este horizonte (cf. infra).

Otra cuestión estrictamente relacionada con la anterior que queda por valorar es la de la pervivencia de la necrópolis más allá de las fechas proporcionadas por los elementos griegos y de su incorporación en la esfera administrativa de Roma, denunciada por la presencia de materiales de cronología republicana (Delgado, 1971; Fabião, 1998, 354; Gomes y Alves, 2017). Como veremos, la discusión de los materiales estudiados en este trabajo y, más genéricamente, de la cerámica griega del OSM permite realizar algunas consideraciones relevantes sobre éstas y otras cuestiones.

2. LA CERÁMICA GRIEGA DE LA NECRÓPOLIS DE ALCÁCER DO SAL: ALGUNOS ASPECTOS DE LA HISTORIA DE LA INVESTIGACIÓN.

La cerámica griega ha constituido, desde el descubrimiento del OSM en el siglo XIX, uno de los elementos más llamativos de la cultura material de dicha necrópolis. Se puede incluso afirmar que a ella se debe, al menos en una primera etapa, la notoriedad adquirida por el yacimiento entre la comunidad científica portuguesa e internacional.

De hecho, desde la primera divulgación de los hallazgos en la necrópolis efectuada por J. N. Possidónio da Silva (1875), los vasos griegos de Alcácer han constituido el epicentro de una activa y en ocasiones encendida discusión, centrada en un primer momento en la autenticidad de las propias piezas y luego, hasta bien avanzado el siglo XX, en la identificación concreta de su procedencia y de sus centros productores (Pereira, 1962, 1-10). 
No obstante, y como en tantos otros aspectos, sería sólo la intervención del Profesor Virgílio Correia la que permitiría establecer de forma sólida las coordinadas para el estudio de este conjunto. El Profesor de Coimbra, haciendo justicia al sentido práctico que con frecuencia se intuye de sus observaciones, ha obtenido directamente del Profesor John Beazley, una de las más reputadas, si no la más reputada autoridad en cerámica griega, su opinión sobre las piezas de Alcácer, y la confirmación del erudito británico de que correspondían a producciones áticas fechables en la primera mitad del siglo IV a.n.e. (Correia, 1925a, 192).

Sin embargo, la primera publicación extensiva y metódica de las piezas de Alcácer no tendrá lugar hasta los años 1960, cuando M. ${ }^{a}$ H. da Rocha Pereira, en su seminal trabajo sobre la cerámica griega en Portugal, estudia por primera vez de forma científica los recipientes de figuras rojas de la necrópolis (Pereira, 1962, 72-84 y 95), estudio al cual vendrían a sumarse en los años siguientes nuevos datos aportados por distintos investigadores (Delgado, 1971; Ferreira, 1971, 316; Rouillard, 1975; MacPhee y Trendall, 1987, 39 y nn. 69-72).

A pesar de estos importantes avances, la publicación completa y global del conjunto, incluyendo al parecer los materiales recogidos en nuevas campañas de excavación dirigidas por A. Cavaleiro Paixão (1970 y 1983), sólo se producirá ya a finales de la década de 1980, de la mano de P. Rouillard y sus colaboradores (Rouillard et al., 1988-9). El trabajo de este equipo, que recoge un total de 42 recipientes de figuras rojas y de barniz negro, ha permitido en efecto realizar por primera vez un inventario completo de la cerámica ática del OSM, así como establecer de forma exacta sus coordinadas técnicas, decorativas y cronológicas.

Desde la publicación de este importante estudio, y a pesar del enorme desarrollo del conocimiento sobre el comercio griego en el actual territorio portugués (Arruda, 1995; 1997; 2006; Arruda, Barros y Lopes 1998; Arruda y Lopes 2012), no se han verificado demasiadas alteraciones al panorama disponible sobre el conjunto de Alcácer do Sal.

Así, el único dato significativo aportado, hasta el momento, por las revisiones posteriores a los años 1980 ha sido la identificación en los fondos del Museo Nacional de Arqueología de un interesante recipiente de figuras negras, clasificado y estudiado por A. M. Arruda (2006, 135-6). De acuerdo con esta investigadora, dicho recipiente corresponde a una "band-cup" del Tipo C de J. Beazley con decoración floral, encuadrable en lo que el investigador británico designó como "floral band-cups" (idem, con bibliografía), y como tal fechable en la primera mitad del siglo $\mathrm{V}$ a.n.e. (ibidem).

3. CERÁMICAS ÁTICAS INÉDITAS DEL OSM: CLASIFICACIÓN, CARACTERIZACIÓN Y SIGNIFICADO.

Si bien es cierto que los excelentes estudios de M ${ }^{a}$. H. da Rocha Pereira y, sobre todo, de P. Rouillard y sus colaboradores, a los que habría que sumar las contribuciones posteriores de A. M. Arruda (1997, 89-90; 2006), han permitido conocer con gran detalle el conjunto de cerámicas áticas de la necrópolis de Alcácer do Sal, no todos los materiales recogidos en distintas épocas y de distintos modos en este conjunto funerario han conocido un tratamiento tan profundo y sistemático.

Así, y considerando que hoy por hoy esos materiales son prácticamente la única fuente fiable para la reconstrucción de la secuencia cultural y cronológica del yacimiento, he acometido en el marco de mi proyecto de Doctorado el estudio integral de la cultura material del OSM, buscando integrarla siempre que fuera posible con la escasa documentación de campo disponible y con las descripciones proporcionadas por los responsables de los distintos episodios de excavación que han tenido lugar en la necrópolis (Gomes, 2016).

Una de las colecciones que he podido estudiar, custodiada por la Dirección Regional de Cultura de Alentejo y depositada en sus instalaciones de Alcácer do Sal, se componía de material todavía por tratar recuperado en las últimas intervenciones de $\mathrm{A}$. Cavaleiro Paixão. El tratamiento y estudio integral de este conjunto me han permitido identificar, además de una serie de otros elementos de gran interés, un pequeño pero significativo conjunto de cerámicas griegas inéditas (Fig. 2 y Tabla 1), que vienen a ampliar el conjunto conocido hasta el momento, reforzando algunas de las tendencias que ya se detectaban en los estudios referidos con anterioridad.

Este conjunto integra ocho fragmentos de cerámica ática correspondientes a otros tantos recipientes inéditos. Entre estos fragmentos, tan sólo uno (Fig. 2, n. 1) corresponde a una pieza de figuras rojas, y específicamente a un borde en pestaña pendiente perteneciente a un plato de pescado, decorado, como el resto de piezas del mismo tipo exhumadas en el OSM (Rouillard et al., 1988-9, 73-5), con un motivo de olas encrespadas. Aunque el frag- 

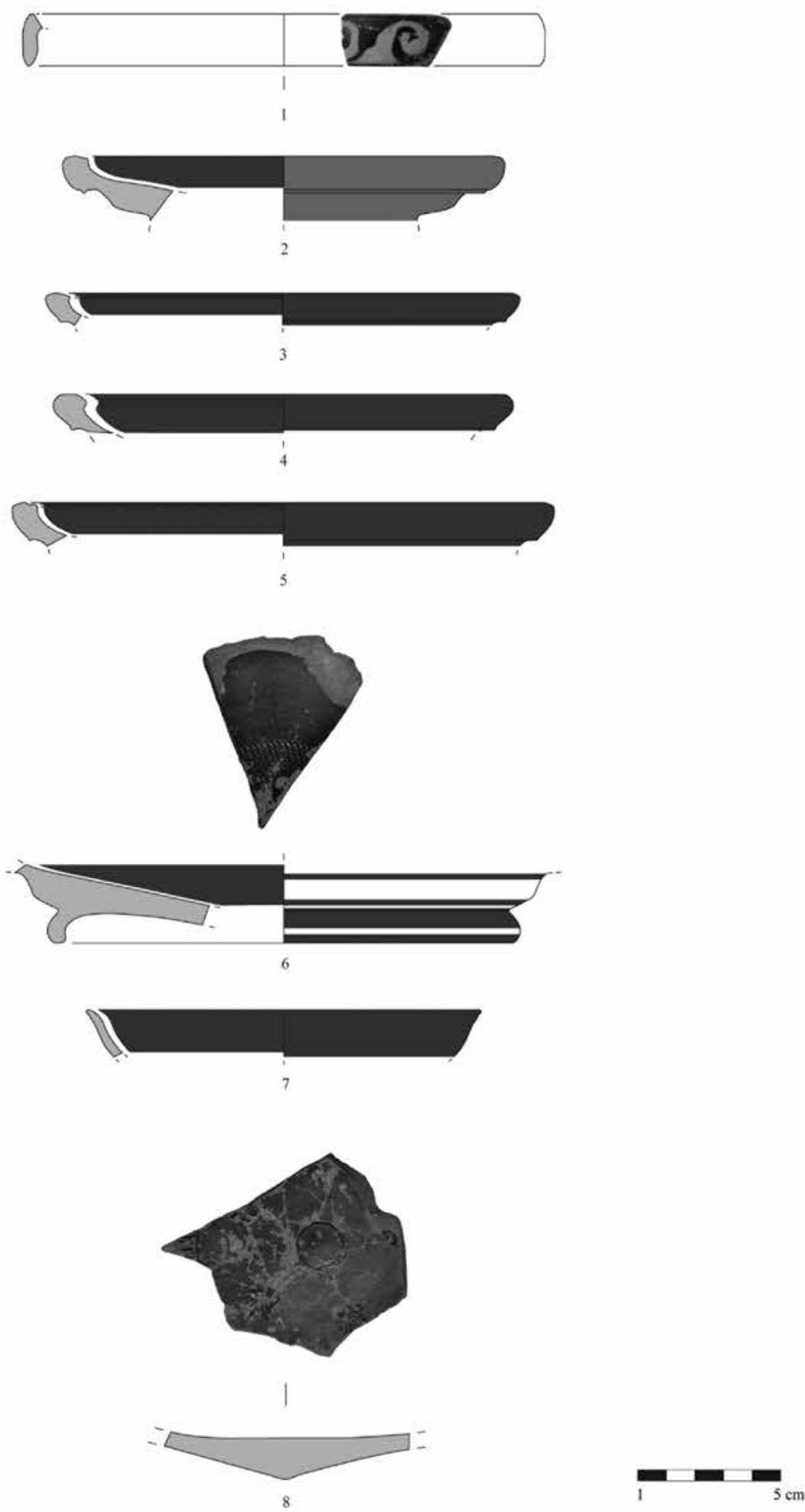

Figura 2. Cerámicas áticas inéditas de la necrópolis de Olival do Senhor dos Mártires. 


\begin{tabular}{|c|c|c|c|c|}
\hline$\#$ & Estilo & Tipologia & $\varnothing$ Borde & $\varnothing$ Fondo \\
\hline 1 & Figuras Rojas & Plato de pescado & $18 \mathrm{~cm}$ & $*$ \\
\hline 2 & Barniz Negro & Rolled rim plate & $15,4 \mathrm{~cm}$ & $*$ \\
\hline 3 & Barniz Negro & Rolled rim plate & $16,6 \mathrm{~cm}$ & $*$ \\
\hline 4 & Barniz Negro & Rolled rim plate & $16 \mathrm{~cm}$ & $*$ \\
\hline 5 & Barniz Negro & Rolled rim plate & $19 \mathrm{~cm}$ & $*$ \\
\hline 7 & Barniz Negro & Rolled rim plate & $*$ & $16 \mathrm{~cm}$ \\
\hline 8 & Barniz Negro & Kylix & $13,8 \mathrm{~cm}$ & $*$ \\
\hline & Barniz Negro & Outturned rim bowl? & $*$ & $*$ \\
\hline
\end{tabular}

Tabla 1. Descripción general de las piezas áticas inéditas de la necrópolis de OSM.

mento en estudio sea muy reducido, creo que puede aceptarse que corresponderá, como sus congéneres ya publicados, a una obra del Pintor de Alcácer do Sal definido por I. MacPhee y A. D. Trendall (1987, nn. 69-72). Esta pieza puede fecharse, como las restantes, de c.380-370 a.n.e..

Los siete fragmentos restantes que he podido identificar corresponden a vajillas de barniz negro, entre los cuales se puede destacar la presencia de cuatro bordes (Fig. 2, nn. 2-5) pertenecientes a platos del tipo "rolled rim plate"de la Ágora de Atenas (Sparkes y Talcott, 1970, 147), también conocidos como Tipo Jehasse 116 (Jehasse y Jehasse, 1973, 357-364). A estos fragmentos clasificables se puede todavía sumar un fondo (Fig. 2, n.6) relativamente bien conservado que presenta un círculo de decoración a ruedecilla y que puede igualmente haber pertenecido a un recipiente de esta misma morfología.

Este tipo de plato se hallaba ya bien representado en la necrópolis, al haberse recogido en el estudio de P. Rouillard y colaboradores cuatro ejemplares con esta morfología (Rouillard et al., 1988-9, 77-8). Estos platos, aunque poco frecuentes fuera de Grecia, parecen haber circulado en cierta cantidad, como se desprende de su abundancia en el pecio de El Sec, en las inmediaciones de Mallorca (Cerdà, 1987, 357-364), hallándose además bien representados en otros conjuntos portugueses, en particular en la región de Algarve, donde se ha documentado su ocurrencia en Castro Marim (Arruda, 1997, 1356) y quizás en Tavira (Barros, 2005).

La cronología de los ejemplares aquí estudiados debe, por otro lado, considerarse idéntica a la de las piezas anteriormente publicadas, y que se han fe- chado en el primer cuarto del siglo IV a.n.e. (Rouillard et al., 1988-9, 77-8).

Otro fragmento que he podido documentar corresponde al borde de una kylix (Fig. 2, n. 7), probablemente encuadrable en la clase de las "stemless cups" de la Ágora de Atenas (Sparkes y Talcott, 1970, 98-105), forma que está bien representada tanto en el mismo OSM (Rouillard et al., 1988-9, 72-3) como más generalmente en los conjuntos de cerámica ática del sur peninsular (Rouillard, 1991; Sánchez, 1991; Domínguez y Sánchez, 2007).

La cronología de esta pieza no es fácil de establecer, ya que le falta el pie, elemento cuya evolución reviste, en las "stemless cups", un importante significado cronológico; así, la pieza en estudio podría fecharse, al menos teóricamente, dentro de un amplio intervalo que va desde la segunda mitad del siglo $\mathrm{V}$ a.n.e. hasta el primer cuarto del siglo IV a.n.e. (Sparkes y Talcott, 1970, 98-105).

Finalmente, el último fragmento inédito que he tenido ocasión de identificar (Fig. 2, n. 8) corresponde al fondo de un recipiente de difícil integración morfológica, que merece no obstante mención al presentar en su faceta interna un esquema decorativo relativamente bien preservado, que consiste en un círculo central inciso rodeado por una corona de palmetas impresas conectadas por trazos igualmente incisos. Estas palmetas pueden integrarse sin dificultad en el Tipo D definido para el material de barniz negro de la necrópolis de Aléria (Jehasse y Jehasse, 1973, Pl. 78, nn.1400-1).

Este motivo central parece, por otro lado, haber estado encuadrado por una moldura de óvulos impresos demarcada por líneas incisas. Estos distintos 
elementos decorativos podrían aproximar la pieza en análisis de las producciones del Taller 5 del pecio de El Sec (Cerdà, 1987, 209 y Lám. II), aunque en dicho taller presenten combinaciones algo distintas de la que se documenta en la pieza portuguesa.

Todos estos elementos podrían sugerir, sin seguridad, que este fondo haya pertenecido a un recipiente del tipo de las "outturned rim bowls" de la Ágora de Atenas (Sparkes y Talcott, 1970, 128-130), forma sobre la cual se aplican dichos elementos y esquemas decorativos, y que se encuentra además bien representada en la necrópolis de Alcácer do Sal (Rouillard et al., 1988-9, 30-32).

Con la inclusión de estos ejemplares inéditos, el conjunto de la cerámica ática del OSM pasa a sumar un total de 50 individuos, que corresponden, de acuerdo con el reciente estudio integral de los materiales cerámicos que he podido realizar, a un 7,5\% del conjunto cerámico total de la Edad del Hierro.

Dentro de esta muestra, y como hemos visto, el estilo de figuras negras se encuentra representado por un único ejemplar, correspondiendo la larga mayoría del conjunto a producciones del horizonte clásico tardío, dentro de las cuales se contabilizan 26 recipientes de figuras rojas y 23 de barniz negro.

No me detendré en este contexto en la descripción sistemática de las características de este conjunto que, salvo las de los materiales inéditos que ahora se presentan, se encuentran muy bien detalladas en otros estudios que he citado ya en varias ocasiones. Así, y a efectos de la discusión que buscaré desarrollar en el siguiente apartado, me he limitado en esta ocasión a presentar en la Tabla 2 las características generales del conjunto, remitiendo a sus respectivas publicaciones.

4. SigNiFICADO COMERCIAL, CULTURAL Y CRONOLÓGICO DE LA CERÁMICA ÁTICA DEL OSM: ALGUNAS REFLEXIONES FINALES.

Aunque haya sido ya estudiado con gran profundidad en distintas ocasiones (Rouillard et al., 1988-9; Rouillard, 1991; Arruda, 1997, 89-90), creo que el conjunto de cerámica griega de la necrópolis del OSM merece en este contexto algunas consideraciones adicionales que sirvan sobre todo para contextualizar el nuevo material que ahora se presenta.

Desde luego, y del punto de vista del significado comercial y económico de este conjunto, me parece relevante señalar que el OSM, aunque constituya en muchos aspectos un ejemplo típico de los ritmos de importación de los productos griegos documenta- dos en al actual territorio portugués, presenta también en otros dominios una innegable especificidad merecedora de atención y discusión.

Desde luego, y en este dominio, no puede dejar de referirse de nuevo la presencia en la necrópolis de un ejemplar decorado con el estilo de figuras negras (Arruda, 2006), ya comentado, que constituye sin duda la más antigua importación de cerámica griega hasta el momento documentada en el Bajo Sado, encuadrándose en un período en el cual la importación de cerámica ática para el Extremo Occidente Peninsular se encuentra sumamente mal documentada.

De hecho, y como es bien sabido, la importación masiva y sistemática de cerámicas griegas no se verifica, en el Occidente Peninsular, hasta mediados, $\mathrm{y}$ sobre todo finales del siglo $\mathrm{V}$ a.n.e., alcanzando su apogeo durante la primera mitad del IV a.n.e. (Arruda, 1995, 1997 y 2006; Rouillard, 1991). El conjunto de recipientes de figuras rojas y de barniz negro del OSM puede considerarse, en este aspecto, bastante paradigmático, siguiendo de forma muy próxima los ritmos globales de importación que pueden, con los datos actuales, restituirse para el resto del territorio portugués (Fig. 3).

Sin embargo, y en cuanto a la composición formal, el conjunto de cerámica griega del OSM ofrece ciertas peculiaridades dignas de mención. Desde luego, llama la atención la diversidad tipológica del material, muy superior a la de los restantes conjuntos portugueses, con la excepción de Castro Marim (Arruda, 1997). De hecho, P. Rouillard ya había señalado este hecho al considerar la necrópolis de Alcácer como un ejemplo de lo que designó como "repertorio amplio de formas", en contraste con la situación verificada en la mayoría de los yacimientos del sur peninsular que estudió (Rouillard, 1991, 191 y carte 14).

A pesar de esa gran diversidad, se nota en el OSM la presencia de un núcleo compuesto por lo que el investigador francés designó como «servicio andaluz», compuesto por el krater de campana y por las kylikes, asociación particularmente característica del mundo Ibérico de la Alta Andalucía (idem: 184).

El paralelismo de una parte sustancial del conjunto de Alcácer do Sal con dicho "servicio» no se limita, además, a los aspectos morfológicos, ya que también en la necrópolis portuguesa se registra la asociación entre obras del Pintor del Tirso Negro y del Grupo de Viena 116, igualmente característica de los repertorios del área Ibérica meridional ( $i b i$ - 


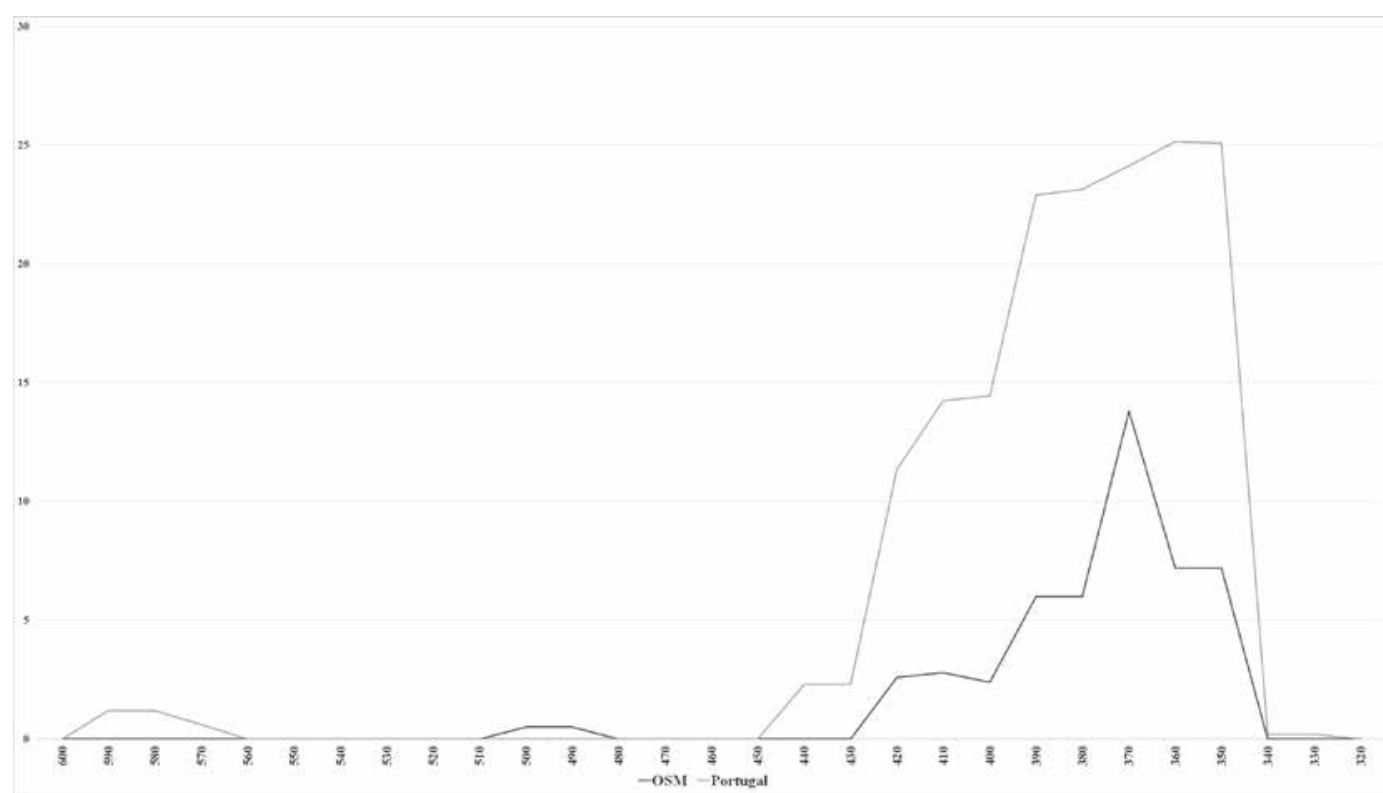

Figura 3. Ritmo de la importación de cerámica griega en la necrópolis de Olival do Senhor dos Mártires y en el restante territorio portugués (número medio de individuos por década, calculado sobre el número mínimo de individuos).

dem). Este detalle merece cierta atención, ya que, como he podido constatar en el estudio integral del material del OSM, dicha similitud cuadra bien con cierta convergencia entre la necrópolis de Alcácer do Sal y las del área cultural Ibérica durante la II Edad del Hierro (Gomes, 2016, 342-3).

Si este «servicio andaluz» constituye un patrón bien conocido, su asociación con otras formas, consideradas en muchos casos como poco comunes, es una especificidad de Alcácer do Sal. Se puede realzar, a este propósito, la gran popularidad de formas como los "rolled rim plates" o, sobre todo, los platos de pescado, siendo esta última forma bastante rara en los inventarios de la cerámica ática de los yacimientos peninsulares (idem, 191), con la excepción de los de la Comunidad Valenciana (García i Martín, 1999; 2000).

Estas observaciones no significan que el conjunto del OSM no pueda considerarse esencialmente homogéneo: lo es, tanto del punto de vista funcional, con un absoluto predominio de los recipientes destinados en su origen a la preparación y consumo del vino y al consumo de alimentos, como también del punto de vista cronológico, al centrarse esencialmente en la primera mitad del siglo IV a.n.e., pudiendo incluso identificarse en su seno distintas series tipificadas de recipientes que bien pueden haber constituido remesas simultáneas (Rouillard, 1991, 185).
Otro aspecto que merece algunos comentarios es la utilización dada a estos recipientes en el yacimiento en estudio. Como antes he podido comentar, las formas atestadas en el OSM corresponden a recipientes para el consumo de bebida y comida; sin embargo, al menos una parte de dichos recipientes parece haber sido reinterpretado localmente, en la medida en que los testimonios del Profesor V. Correia, que ha podido exhumar algunas de estas piezas in situ, comprueban su utilización como contenedores cinerarios. El Profesor de Coimbra señala, por ejemplo, que “... na sepultura n.. 8 havia um bojudo vaso globular junto de duas crateras de tipo oxibaphon, tôdas contendo ossos" (Correia, $1928,173)$, con lo cual parece claramente atestado el uso de los krateres como urnas. Siempre siguiendo los testimonios del mismo investigador, otros recipientes, como un skyphos de la misma tumba n. ${ }^{\circ} 8$, podrán haberse depositado como vasos de ofrendas.

El uso del krater como urna cineraria, caso prácticamente único hasta el momento en el territorio portugués (v., no obstante, infra), no es, aun así, una especificidad de la necrópolis de Alcácer, ya que la misma práctica parece haber sido corriente, una vez más, en el mundo Ibérico, y en particular en el área Bastetana de la Alta Andalucía (Rouillard, 1991, 180; y 2009, 366), con lo cual parece representar una curiosa homología más entre el OSM y el mundo funerario de aquella área cultural. 


\begin{tabular}{|c|c|c|c|c|}
\hline Forma & Estilo & Pintor & Cronología & Bibliografía \\
\hline Band cup & $\mathrm{FN}$ & $\begin{array}{c}\text { Indeterminado } \\
\text { (Grupo das floral band-cups) }\end{array}$ & $500-475$ & Arruda 2006 \\
\hline Peliké & $\mathrm{FV}$ & Círculo do Pintor do Tirso Negro & $375-350$ & $\begin{array}{l}\text { Pereira 1962; Trías de Arribas 1967; } \\
\text { Rouillard et al. 1988-9 ; Arruda } 1997\end{array}$ \\
\hline Peliké & $\mathrm{FV}$ & Indeterminado & $375-350$ & Rouillard et al. 1988-9 ; Arruda 1997 \\
\hline Peliké & FV & Indeterminado & $375-350$ & Rouillard et al. 1988-9 ; Arruda 1997 \\
\hline Krater de sino & $\mathrm{FV}$ & Grupo de Viena 1025 & $400-375$ & $\begin{array}{l}\text { Pereira 1962; Trías de Arribas 1967; } \\
\text { Rouillard et al. 1988-9 ; Arruda } 1997\end{array}$ \\
\hline Krater de sino & FV & Grupo de Viena 1025 & $400-375$ & Rouillard et al. 1988-9 ; Arruda 1997 \\
\hline Krater de sino & $\mathrm{FV}$ & Pintor de Telos & $380-370$ & Rouillard et al. 1988-9 ; Arruda 1997 \\
\hline Krater de sino & $\mathrm{FV}$ & Pintor do Tirso Negro & $375-350$ & $\begin{array}{l}\text { Pereira 1962; Trías de Arribas 1967; } \\
\text { Rouillard et al. 1988-9 ; Arruda } 1997\end{array}$ \\
\hline Krater de sino & FV & Pintor do Tirso Negro & $375-350$ & $\begin{array}{c}\text { Rouillard 1975; Rouillard et al. 1988-9; } \\
\text { Arruda } 1997\end{array}$ \\
\hline Krater de sino & FV & Pintor do Tirso Negro & $375-350$ & Rouillard et al. 1988-9 ; Arruda 1997 \\
\hline Krater de sino & FV & Indeterminado & $375-350$ & Rouillard et al. 1988-9 ; Arruda 1997 \\
\hline Krater de sino & FV & Indeterminado & $375-350$ & Rouillard et al. 1988-9 ; Arruda 1997 \\
\hline Krater de sino & $\mathrm{FV}$ & Indeterminado & $400-350$ & Rouillard et al. 1988-9 ; Arruda 1997 \\
\hline Krater de sino & $\mathrm{FV}$ & Indeterminado & $400-350$ & Rouillard et al. 1988-9 ; Arruda 1997 \\
\hline Stemless cup & FV & Grupo de Viena 116 & $375-350$ & Rouillard et al. $1988-9$; Arruda 1997 \\
\hline Stemless cup & $\mathrm{FV}$ & Grupo de Viena 116 & $375-350$ & Rouillard et al. 1988-9 ; Arruda 1997 \\
\hline Stemless cup & $\mathrm{FV}$ & Grupo de Viena 116 & $375-350$ & Rouillard et al. 1988-9 ; Arruda 1997 \\
\hline Stemless cup & FV & Indeterminado & $375-350$ & Rouillard et al. 1988-9 ; Arruda 1997 \\
\hline Skyphos & FV & Grupo del Fat Boy & $375-350$ & $\begin{array}{l}\text { Pereira 1962; Rouillard et al. 1988-9 ; } \\
\text { Arruda } 1997\end{array}$ \\
\hline Skyphos & FV & Grupo del Fat Boy & $375-350$ & $\begin{array}{c}\text { Pereira 1962; Rouillard et al. 1988-9 ; } \\
\text { Arruda } 1997\end{array}$ \\
\hline Prato de peixe & $\mathrm{FV}$ & Pintor de Alcácer do Sal & $380-370$ & $\begin{array}{l}\text { Pereira 1962; Trías de Arribas 1967; Frel } \\
\text { 1969; MacPhee / Trendall 1987; Rouillard } \\
\text { et al. 1988-9 ; Arruda } 1997\end{array}$ \\
\hline Prato de peixe & $\mathrm{FV}$ & Pintor de Alcácer do Sal & $380-370$ & $\begin{array}{l}\text { MacPhee / Trendall 1987; Rouillard et al. } \\
\text { 1988-9; Arruda } 1997\end{array}$ \\
\hline Prato de peixe & FV & Pintor de Alcácer do Sal & $380-370$ & $\begin{array}{l}\text { MacPhee / Trendall 1987; Rouillard et al. } \\
\text { 1988-9; Arruda } 1997\end{array}$ \\
\hline Prato de peixe & $\mathrm{FV}$ & Pintor de Alcácer do Sal & $380-370$ & $\begin{array}{l}\text { MacPhee / Trendall 1987; Rouillard et al. } \\
\text { 1988-9; Arruda } 1997\end{array}$ \\
\hline Prato de peixe & $\mathrm{FV}$ & Pintor de Alcácer do Sal? & $380-370$ & Rouillard et al. 1988-9 ; Arruda 1997 \\
\hline
\end{tabular}




\begin{tabular}{|c|c|c|c|c|}
\hline Prato de peixe & FV & Pintor de Alcácer do Sal? & $380-370$ & Rouillard et al. $1988-9$; Arruda 1997 \\
\hline Prato de peixe & $\mathrm{FV}$ & Pintor de Alcácer do Sal? & $380-370$ & Material inédito \\
\hline Stemless cup & VN & N/A & $450-375 ?$ & Material inédito \\
\hline Bolsal & VN & N/A & $375-350$ & Rouillard et al. 1988-9 ; Arruda 1997 \\
\hline Bolsal & VN & N/A & $375-350$ & Rouillard et al. 1988-9 ; Arruda 1997 \\
\hline Bolsal & VN & N/A & $425-400$ & Rouillard et al. 1988-9 ; Arruda 1997 \\
\hline $\begin{array}{l}\text { Outturned rim } \\
\text { bowl }\end{array}$ & VN & N/A & $400-375$ & $\begin{array}{l}\text { Ferreira 1971; Rouillard et al. 1988-9; } \\
\text { Arruda } 1997\end{array}$ \\
\hline $\begin{array}{l}\text { Outturned rim } \\
\text { bowl }\end{array}$ & VN & $\mathrm{N} / \mathrm{A}$ & $400-375$ & Rouillard et al. 1988-9 ; Arruda 1997 \\
\hline $\begin{array}{l}\text { Outturned rim } \\
\text { bowl }\end{array}$ & VN & N/A & $400-350$ & Rouillard et al. 1988-9 ; Arruda 1997 \\
\hline $\begin{array}{l}\text { Outturned rim } \\
\text { bowl? }\end{array}$ & VN & N/A & $400-350$ & Material inédito \\
\hline Saltcellar & VN & $\mathrm{N} / \mathrm{A}$ & $400-350$ & Rouillard et al. 1988-9 ; Arruda 1997 \\
\hline Saltcellar & VN & N/A & $400-350$ & Rouillard et al. 1988-9 ; Arruda 1997 \\
\hline Taça, tipo ind. & VN & N/A & $400-350$ & $\begin{array}{c}\text { Delgado 1971; Rouillard et al. 1988-9; } \\
\text { Arruda } 1997\end{array}$ \\
\hline Taça, tipo ind. & VN & N/A & $400-350$ & Rouillard et al. 1988-9 ; Arruda 1997 \\
\hline Taça, tipo ind. & VN & N/A & $400-350$ & Rouillard et al. 1988-9 ; Arruda 1997 \\
\hline Taça, tipo ind. & VN & N/A & $400-350$ & Rouillard et al. 1988-9 ; Arruda 1997 \\
\hline Rolled rim plate & VN & N/A & $400-375$ & $\begin{array}{c}\text { Delgado 1971; Rouillard et al. 1988-9; } \\
\text { Arruda } 1997\end{array}$ \\
\hline Rolled rim plate & VN & $\mathrm{N} / \mathrm{A}$ & $400-375$ & Rouillard et al. 1988-9 ; Arruda 1997 \\
\hline Rolled rim plate & VN & $\mathrm{N} / \mathrm{A}$ & $400-375$ & Rouillard et al. 1988-9 ; Arruda 1997 \\
\hline Rolled rim plate & VN & N/A & $400-375$ & Rouillard et al. 1988-9 ; Arruda 1997 \\
\hline Rolled rim plate & VN & N/A & $400-375$ & Material inédito \\
\hline Rolled rim plate & VN & N/A & $400-375$ & Material inédito \\
\hline Rolled rim plate & VN & N/A & $400-375$ & Material inédito \\
\hline Rolled rim plate & VN & N/A & $400-375$ & Material inédito \\
\hline Rolled rim plate? & VN & N/A & $400-375$ & Material inédito \\
\hline
\end{tabular}

Tabla 2. Características globales del conjunto de cerámica ática de la necrópolis de OSM 
No obstante, debe señalarse que la aparente excepcionalidad de este uso funerario de la cerámica griega del OSM, que obliga a buscar paralelos en áreas relativamente lejanas, puede resultar tan sólo de un persistente vacío de información sobre las prácticas funerarias de la II Edad del Hierro en la Baja Andalucía y en el área meridional portuguesa.

De hecho, el reciente estudio de dos vasos griegos de la necrópolis de Cerro Furado (Baleizão, Beja) - un krater de campana del Pintor del Tirso Negro y un skyphos, probablemente del Grupo del Fat Boy (Arruda y Lopes, 2012), idénticos, como tales, a algunas piezas de Alcácer - podría estar indicando que el tipo de utilización de piezas griegas como urnas y/o recipientes de ofrendas que se puede documentar en el OSM habrá tenido una mayor difusión en el suroeste peninsular de la que actualmente se conoce. A este respecto, no puede también dejar de recordarse la deposición de piezas de barniz negro - en este caso, dos "outturned rim bowls" en una tumba de incineración de la necrópolis de la II Edad del Hierro de Quinta da Queimada (Lagos) (Calado y Gomes, 2006).

El restante repertorio documentado, que se relaciona, como ya he podido comentar, con el consumo de la bebida y de la comida, puede, por otro lado, haber constituido el equipamiento privilegiado de banquetes funerarios realizados en el momento de la deposición de los muertos o haber servido, alternativamente, para realizar libaciones y ofrendas funerarias (Rouillard, 2009, 366).

No obstante, debo subrayar que no me parece suficientemente comprobado que la intención primaria subyacente a la importación de estos recipientes fuera específicamente funeraria. Creo, en efecto, que los datos actuales no permiten excluir - pero tampoco, es cierto, comprobar - que esta cerámica de lujo haya desempeñado, en un primer momento, una función más ajustada a su uso original, concretamente en el marco de banquetes y symposia que podrán haber servido a las élites locales para proyectar y legitimar su ascendencia social, contribuyendo simultáneamente a la construcción de la identidad del grupo.

De hecho, la práctica del banquete y del comensalismo se encuentra bien documentada en la Protohistoria Peninsular (Armada Pita, 2008; Grau Mira, 2010; Sardá Seuma, 2010) y, de forma más particular, en la Edad del Hierro del Suroeste (Berrocal Rangel 2004; Celestino y Cabrera, 2008;
Gomes, 2014a, 39), donde el ejemplo del Altar A de Castrejón de Capote (Berrocal Rangel, 1994 y 2004) demuestra la importancia de este tipo de prácticas colectivas ritualizadas todavía durante las etapas avanzadas de la Edad del Hierro.

Por otra parte, la presencia en la necrópolis y en el mismo poblado de ralladores de tipo etrusco (Arruda et al., e. p.), asociados a menudo a la aromatización del vino con queso (Ridgway, 1997), podría invocarse como argumento a favor de la presencia de este tipo de prácticas en la Alcácer do Sal prerromana.

La utilización funeraria de los recipientes griegos que vengo comentando podría así revestirse de un sentido adicional, al no constituir solamente un bien de prestigio per se, sino además un elemento con un sentido social y político añadido localmente cuya amortización final constituiría un recurso más en la construcción de discursos de poder perfectamente articulados.

Esta hipótesis, aunque sea conjetural - sólo la publicación de nuevos contextos, en particular correspondientes al poblado, podría permitir evaluarla de forma más concreta - conlleva no obstante un problema adicional relativo a la cronología de las piezas en estudio. Hemos visto ya que sus fechas de producción, aparte de bien establecidas, presentan una notable homogeneidad; sin embargo, en la ausencia de contextos arqueológicos fiables para estas piezas, no puede excluirse la existencia de desfases, más o menos importantes, entre las fechas de producción y las de deposición de estas vajillas, sobre todo si se admitiera que dicha deposición pueda haber sido precedida de un período más o menos dilatado de utilización en contextos no-funerarios.

El atesoramiento de vasos griegos y su deposición en contextos incompatibles con su cronología de producción no es del todo un fenómeno desconocido en la Península (Rouillard, 2009, 368-9), y en particular en el mundo Ibérico (García Cano, 1999) - siendo el caso recientemente identificado y estudiado de los siete krateres áticos de la cámara principesca de Píquia, en Jaén, de cronología Romana Republicana, un ejemplo extremo (Ruiz et al., 2015; Rueda y Olmos, 2015) - por lo cual me parece importante tener presente la posibilidad de que hayan ocurrido fenómenos de este tipo, sobre todo a la hora de valorar el significado de estos recipientes para el establecimiento de la secuencia global de la necrópolis. 
De hecho, la cerámica griega del OSM, por su gran precisión cronológica, se ha utilizado, naturalmente, para fechar el horizonte de la segunda Edad del Hierro de la necrópolis, así como para establecer las fechas terminales de su utilización prerromana (Arruda, 1999-2000, 81), ya que los materiales hasta hace poco conocidos y publicados no eran suficientemente expresivos como para avalar la existencia de horizontes de utilización posteriores a c. 350 a.n.e..

Sin embargo, parece importante tener en consideración que la quiebra en la importación de vasos griegos a partir de mediados/tercer cuarto del siglo IV a.n.e. no constituye una particularidad de la necrópolis de Alcácer do Sal, sino más bien un fenómeno generalizado (Rouillard, 1991; Arruda, 1995; 1997). Bien así, no debe perderse de vista que existe en el área estudiada una efectiva escasez de buenos indicadores cronológicos durante la segunda mitad del siglo IV y prácticamente todo el siglo III a.n.e., sólo minimizada en áreas más meridionales por la difusión de las producciones helenísticas de tipo "Kuass" (Niveau, 2003).

Además de estas consideraciones de índole general, puedo afirmar que la revisión integral que he realizado del material exhumado en la necrópolis del OSM me ha permitido identificar ciertos elementos - en particular piezas que imitan o que se inspiran en prototipos griegos o, más genéricamente, helenísticos (Gomes 2016: 195-211) - que pueden contribuir a la definición de un horizonte tardío dentro de la necrópolis, posterior a la fecha de abandono tradicionalmente propuesta.

Por otra parte, me parece muy significativa la presencia en el área del OSM de un conjunto no despreciable de materiales de cronología republicana, más numeroso que lo que estudios anteriores (Delgado, 1971; Fabião, 1998) dejaban suponer, cuya naturaleza parece a todos títulos poder identificarse como efectivamente funeraria (Gomes, 2014b; Gomes y Alves, 2017).

Parece difícil explicar la reactivación en los albores del período romano de una necrópolis abandonada hace dos siglos, siendo en mi opinión mucho más probable que se haya verificado un uso continuado de este espacio, que no obstante parece haber sufrido una fuerte retracción, quizás como resultado de la fundación de otra necrópolis al Norte del castillo de Alcácer do Sal, la de S. Francisco, que se ha identificado y excavado pero que sigue todavía prácticamente inédita (Faria, 2002, 63-4).
El conjunto de la cerámica griega de la necrópolis del OSM, que en la presente contribución busqué ampliar con la adición de nuevos ejemplares que permanecían hasta el momento inéditos, sigue siendo uno de los útiles privilegiados para estudiar el horizonte de la II Edad del Hierro de Alcácer do Sal y del Bajo Sado en general, y de igual modo, como intenté demonstrar en las páginas anteriores, para valorar las relaciones y las afinidades comerciales y culturales de Alcácer do Sal durante esa etapa histórica.

Así, y aunque puedan parecer en ocasiones algo especulativas, creo que todas las consideraciones críticas presentadas en las páginas anteriores merecen ser formuladas y discutidas para lograr un mejor conocimiento de la secuencia de la importante necrópolis de Alcácer y, sobre todo, para sentar las bases de una investigación futura, que involucre también los abundantes datos del poblado todavía en estudio por distintos investigadores.

\section{Agradecimientos}

Me gustaría agradecer a la Profesora Doctora Ana Margarida Arruda, cuyo apoyo en la clasificación y estudio de las piezas estudiadas en este trabajo fue fundamental para su ejecución. Me gustaría igualmente expresar mi gratitud a la Dirección Regional de Cultura de Alentejo por haberme permitido estudiar la colección en la que se incluyen estos materiales, y en particular a la Dr ${ }^{\mathrm{a}}$. Esmeralda Gomes, que me ha prestado un inestimable apoyo en el curso de dicho estudio. Agradezco igualmente a la Dr ${ }^{\mathrm{a}}$. Marisol Ferreira, del Excm..$^{\circ}$ Ayuntamiento de Alcácer do Sal, por todo su apoyo.

\section{Bibliografía}

Armada Pita, X. L. (2008), “¿Carne, drogas o alcohol? Calderos y banquetes en el Bronce Final de la Península Ibérica”, Cuadernos de Prehistoria y Arqueología de la Universidad de Granada, 18, 125-162.

Arruda, A. M. (1995), "Panorama das importações áticas em Portugal", Huelva Arqueológica, 13:1, 129-154.

Arruda, A. M. (1997), As cerâmicas áticas do Castelo de Castro Marim, Lisboa.

Arruda, A. M. (1999-2000), Los Fenicios en Portugal. Fenicios y mundo indigena en el centro y sur de Portugal (siglos VIII-VI a.C.), Barcelona.

Arruda, A. M. (2006), "Cerâmicas gregas encontradas em Portugal”, Vasos Gregos em Portugal 
- Aquém das Colunas de Hércules (Pereira, Ma . H. da R. y Arruda, A. M., coords.), Lisboa, 135-140.

Arruda, A. M., Barros, P. y Lopes, V. (1998), "Cerâmicas áticas de Mértola”, Conímbriga, 37, 122149.

Arruda, A. M., Ferreira, M., Sousa, E. de, Lourenço, P., Lima, J. e Carvalho, A. R. (en prensa), Contributos para o conhecimento da Idade do Ferro de Alcácer do Sal: os dados da Rua do Rato, Alcácer do Sal.

Arruda, A. M. y Lopes, M․ C. (2012), “Dois vasos gregos da necrópole do Cerro Furado (Baleizão, Beja - Portugal)”, O Arqueólogo Português, Série V, 2, 401-415.

Barros, P. (2005), "Cerâmicas Áticas no Circuito do Estreito do Extremo-Ocidente Peninsular: Quinta da Queimada, Ilhéu do Rosário, Faro e Tavira”, El Período Orientalizante (Jiménez Ávila, J. \& Celestino Pérez, S., coords.), Madrid, 931-946.

Berrocal Rangel, L. (1994), El altar prerromano de Castrejón de Capote. Ensayo etnoarqueológico de un ritual céltico en el suroeste peninsular, Madrid.

Berrocal Rangel, L. (2004), "Banquetes y rituales colectivos en el suroeste peninsular", Cuadernos de Prehistoria y Arqueología de la Universidad Autónoma de Madrid, 30, 105-119.

Calado, D. y Gomes, M. V. (2006), "Quinta da Queimada (Lagos): a necrópole da II Idade do Ferro”, Revista Portuguesa de Arqueologia, 9:2, 171-185.

Celestino, S. y Cabrera, A. (2008), "El banquete privado y el banquete comunal en el santuario de Cancho Roano", Cuadernos de Prehistoria y Arqueología de la Universidad de Granada, 18, 189-215.

Cerdà, D. (1987), "La cerámica ática de barniz negro”, El Barco de El Sec (Calvià, Mallorca): estudio de los materiales (ARRIBAS, A., TRIAS, M. G., CERDÀ, D. \& HOZ, J. de), Mallorca, 197-400.

Correia, V. (1925a), "Uma conferência sobre a Necrópole de Alcácer do Sal”, Obras. Volume IV, Estudos Arqueológicos, Coimbra, 151-168.

Correia, V. (1925b), "Fechos de cinturão da Necrópole de Alcácer do Sal”, Obras. Volume IV, Estudos Arqueológicos, Coimbra, 187-195.

Correia, V. (1925c), "Um amuleto egípcio da Necrópole de Alcácer do Sal”, Obras. Volume IV,
Estudos Arqueológicos, Coimbra, 195-201.

Correia, V. (1928), "Escavações realizadas na $\mathrm{Ne}-$ crópole Pré-Romana de Alcácer do Sal em 1926 e 1927", Obras. Volume IV, Estudos Arqueológicos, Coimbra, 169-179.

Correia, V. (1930), “As fíbulas da Necrópole de Alcácer do Sal”, Obras. Volume IV, Estudos Arqueológicos, Coimbra, 181-186.

Delgado, M. (1971), "Cerâmica Campaniense em Portugal”, Actas do II Congresso Nacional de Arqueologia, Coimbra, 403-424.

Dominguez, A. y Sánchez, C (2001), Greek Pottery from the Iberian Peninsula. Archaic and Classical Periods, Leiden.

Fabião, C. (1998), O Mundo Indígena e a sua Romanização na Área Céltica do actual território português. Tesis Doctoral presentada a la Facultad de Letras de la Universidad de Lisboa. Edición policopiada.

Faria, J. C. (2002), Alcácer do Sal ao Tempo dos Romanos, Lisboa.

Ferreira, O. da V. (1971), "Cerâmica negra de tipo grego encontrada em Portugal", Arqueologia e História, III, 313-332.

Frankenstein, S. (1997), Arqueología del colonialismo. El impacto fenicio y griego en el sur de la Península Ibérica y el suroeste de Alemania, Barcelona.

Frel, J. (1969): "Les plats à poisson attiques", Listy filologické, $92: 4,291-293$.

García Cano, J. M. (1999), "Un aspecto poco tratado en las necrópolis ibéricas: la perduración de objetos en los ajuares: el caso de Murcia”, Primeras Jornadas de Arqueología Ibérica en Castilla-La Mancha (Valero Tévar, M. A., coord.), Toledo, 169-178.

García I Martín, J. M. (1999), “Algunas observaciones sobre los platos de pescado áticos en la Península Ibérica", Actas del XXIV Congreso Nacional de Arqueología, Vol. 3, Murcia, 1618.

García I Martín, J. M. (2000), "Els plats de peix grecs: evolució tipològica i distribució a la península ibèrica i les illes balears”, Empúries, 52, 185-200.

Gomes, F. B. (2014a), "Importações mediterrâneas em contextos «Pós-Orientalizantes» do Sul de Portugal (séculos VI-IV a.n.e.)”, Onoba, 2, 2744.

Gomes, F. B. (2014b), "Uma terracota de estilo helenístico da necrópole do Olival do Senhor dos 
Mártires (Alcácer do Sal): breves notas”, Conimbriga, 53, 81-97.

Gomes, F. B. (2015), “The Olival do Senhor dos Mártires necropolis (Alcácer do Sal, Portugal) in the context of the Iron Age funerary practices of the Southwestern Iberian Peninsula", Death as Archaeology of Transition: Thoughts and Materials (Rocha, L., Bueno, P. y Branco, G., eds.), Oxford, 327-341.

Gomes, F. B. (2016), Contactos culturais e discursos identitários na I Idade do Ferro do Sul de Portugal (séculos VIII-V a.n.e.): leituras a partir do registo funerário. Tesis Doctoral presentada a la Facultad de Letras de la Universidad de Lisboa.

Gomes, F. B. y Alves, C. (2017), “The final phases of the Olival do Senhor dos Mártires necropolis (Alcácer do Sal, Portugal): the Roman Republican material", $S P A L, 26,87-111$.

Grau Mira, I. (2010), "Vajillas mediterráneas y prácticas de comensalidad en el área central de la Contestania Ibérica”, De la cuina a la taula. IV Reunió d'Economia en el Primer Milleni $A C$ (Mata, C., Pérez Jordá, G. y Vives-Ferrándiz Sánchez, J., eds.), Valencia, 263-270.

Jehasse, J. y Jehasse, L. (1973), La nécropole préromaine d'Aléria (1960-1968), Paris.

MacPhee, I. y Trendall, A. D. (1987), Greek Redfigured Fish-plates, Basel.

Niveau, A. Mª . (2003), Las cerámicas gaditanas barnizadas de "tipo Kuass". Tipología, producción y distribución, Madrid.

Paixão, A. C. (1970), A necrópole do Senhor dos Mártires, Alcácer do Sal. Novos elementos para o seu estudo. Tesis de Licenciatura presentada a la Facultad de Letras de la Universidad de Lisboa.

Paixão, A. C. (1983), "Uma nova sepultura com escaravelho da necrópole proto-histórica do Senhor dos Mártires, Alcácer do Sal”, O Arqueólogo Português, S. 4, 1, 273-286.

Paixão, A. C. (2014), "A necrópole do Olival do Senhor dos Mártires (Alcácer do Sal). Novos elementos para o seu estudo", Estudos Arqueológicos de Oeiras, 21, 429-460.

Pereira, Ma. H. da R. (1962), Greek Vases in Portugal, Coimbra.

Ridgway, D. (1997), "Nestor's Cup and the Etruscans”, Oxford Journal of Archaeology, 16:3, 325-344.

Rouillard, P. (1975), “Un cratère inédit du Peintre du Thyrse Noire à Alcácer do Sal”, Conímbriga, XIV, 177-183.

Rouillard, P. (1991), Les Grecs et la Péninsule Ibérique : du VIIIe au IVe siècle avant Jésus Christ, Paris.

Rouillard, P. (2009), "Le vase grec entre statut et function: le cas de la Péninsule Ibérique", Shapes and uses of Greek vases (7th-4th centuries B.C.) (Tsingarida, A., ed.), Bruxelas, 366376.

Rouillard, P., Paixão, A. C., Villanueva-Puig, M.C. y Durand, J.-L. (1988-9), "Les vases grecques d'Alcácer do Sal”, O Arqueólogo Português, S.4, 6-7, 43-108.

Rueda, C. \& Olmos, R. (2014), "Las crateras áticas de la Cámara Principesca de Piquía (Arjona): los vasos de la memoria de uno de los últimos linajes iberos”, Jaén, Tierra Ibera. 40 Años de Investigación y Transferencia (Ruiz, A. y Molinos, M., eds.), Jaén, 375-392.

Ruiz, A., Molinos, M., Rísquez, C., Gómez, F. y Ángel, M. (2014), "La cámara de Píquia (Arjona)", Jaén, Tierra Ibera. 40 Años de Investigación y Transferencia (Ruiz, A. y Molinos, M., eds.), Jaén, 357-374.

Sánchez, C. (1991), El comercio de productos griegos en Andalucía oriental siglos Vy IV a. C. Estudio tipológico e iconográfico de la cerámica. Tesis Doctoral presentada a la Universidade Complutense de Madrid.

Sardá Seuma, S. (2010), "El giro comensal: nuevos temas y nuevos enfoques en la Protohistoria peninsular", Herakleion, 3, 37-65.

Silva, J. P. N. da (1875), "Uma necrópolis romana em Portugal”, Boletim da Real Associação dos Architectos Civis e Archeologos Portugueses, S.I, 6, 91.

Silva, C. T. da, Soares, J., Beirão, C. de M., Dias, L. F. y Coelho-Soares, A. (1980-1), "Escavações arqueológicas no Castelo de Alcácer do Sal (campanha de 1979)”, Setúbal Arqueológica, 6-7, 149-218.

Sparkes, B. A. y Talcott, L. (1970), The Athenian Agora, Volume XII. Black and plain pottery of the 6th, 5th and 4th centuries B.C., Princeton.

Trías de Arribas, G. (1967), Cerámicas griegas de la Península Ibérica, Valencia. 